\title{
THE INVERSE THEOREM OF APPROXIMATION THEORY IN SMIRNOV-ORLICZ CLASSES
}

\author{
SADULLA Z. JAFAROV
}

Abstract. Let $\Gamma$ be a Dini- smooth curve in the complex plane $\mathbb{C}$. In this study we prove inverse theorem of approximation theory by polynomials in Smirnov-Orlicz classes $E_{M}(G)$.

Mathematics subject classification (2010): 30E10, 41A10, 41A25, 46E30.

Keywords and phrases: Dini-smooth curve, Cauchy singular operator, Faber polynomials, inverse theorem, Orlicz space, Boyd indices, modulus of continuity.

\section{REFERENCES}

[1] S. JA. AL'PER, Approximation in the Mean of Analytic functions of Class $E_{p}$, Gos. Izdat. Fiz.-Mat. Lit., Moscow, 1960, 272-286 (in Russian).

[2] J. E. Andersson, On the degree of polynomial approximation in $E^{p}(D)$, J. Approx. Theory 19 (1977), 61-68.

[3] M. I. ANDRASKO, On the approximation in the mean of analytic functions in regions with smooth boundaries, Problems in mathematical physics and function theory, Izdat. Akad. Nauk. Ukrain. RSR, Kiev, 1963, 1, p. 3 (in Russian).

[4] R. AkgÜn AND D. M. IsRAFllov, Approximation and moduli of fractional orders in Smirnov-Orlicz classes, Glas. Mat. 43, 63 (2008), 121-136.

[5] G. M. GoluZIn, Geometric Theory of Functions of a Complex Variable, Translation of Mathematical Monographs vol. 26, R.I.: AMS, Providence, 1968.

[6] I. I. IBRAGIMOv, D. I. MAMEDKhanov, Constructive characterization of a certain class of functions, Dokl. Akad. Nauk 16 (1976), 820-823.

[7] D. M. IsRAFILOV, Approximation properties of generalized Faber series in an integral metric, Izv. Akad. Nauk Azerb Ser. Fiz.-Tekh. Mat. Nauk 2 (1987), 10-14 (in Russian).

[8] D. M. IsRafiLov, Approximation by p-Faber polynomials in the weighted Smirnov class $E^{p}(G, w)$ and the Bieberbach polynomials, Constr. Approx. 17, 3 (2001), 335-351.

[9] D. M. IsRAFILOV, Approximation by p-Faber Laurent rational functions in the weighted Lebesque spaces, Czechoslovak Math. J. 54, 3(129) (2004), 751-765.

[10] D. M. IsRafilov And A. Guven, Approximation in weighted Smirnov classes, East J. Approx. 11, 1 (2005), 91-102.

[11] D. M. IsRafilov, B. OKtay And R. AKgÜN, Approximation in Smirnov-Orlicz classes, Glas. Mat. 40 (60) (2005), 87-102.

[12] D. M. IsRafilov, Ali Guven, Approximation by trigonometric polynomials in weighted Orlicz spaces, Studia Math. 174, 2 (2006), 147-168.

[13] D. M. IsRafilov AND R. AKGÜN, Approximation in weighted Smirnov-Orlicz classes, J. Math. Kyoto Univ. 46, 4 (2006), 775-770.

[14] S. Z. JAFARov, Approximation by rational functions in Smirnov-Orlicz classes, J. Math. Anal. Appl. 379 (2011), 870-877.

[15] S. Z. JAfarov, S. M. Nikol'skii type inequality and estimation between the best approximation of a functions in norms of different spaces, Math. Balkanica (N.S.) 21, 1-2 (2007), 173-182.

[16] S. Z. JAFARov, Approximations of Harmonic Functions Classes with Singularities on Quasiconformal Curves, Taiwanise J. Math. 12, 3 (2008), 829-840. 
[17] S. Z. JAFAROV, Approximation by polynomials and rational functions in Orlicz spaces, J. Comput. Anal. Appl. (JoCAAA) 13, 5 (2011), 953-962.

[18] M. A. Krasnoselskit And Ya. B. RutickiI, Convex Functions and Orlicz Spaces, P. Norrdhoff Ltd., Groningen, 1961.

[19] V. KokilashVIli, On analytic functions of Smirnov-Orlicz classes, Studia Math. 31 (1968), 43-59.

[20] A. YU. KARLOVICH, Algebras of singular integral operators with piecewise continuous coefficients on reflexive Orlicz spaces, Math. Nachr. 179 (1996), 187-222.

[21] A. YU. KARLOVICH, Algebras of singular integral operators with PC coefficients in rearrangementinvariant spaces with Muckernhoupt weights, J. Operator Theory 47 (2002), 303-323.

[22] W. Matuszewska And W. Orlicz, On certain properties of $\phi$-functions, Bull. Acad. Polon. Sci., Ser. Math. Aster. et Phys. 8-7 (1960), 439-443.

[23] D. I. MAMEDKhanov, Approximation in complex plane and simgular operators with a Cauchy kernel, Dissertation Doct. Phys-math. nauk. The University of Tblisi, 1984 (in Russian).

[24] H. N. MHASKAR, Introduction to the Theory of Weighted Polynomial Approximation, Series in Approximation and Decompositions 7, World Sci., River Edge, NJ, 1996.

[25] CH. Pommerenke, Boundary Behavior of Conformal Maps, Berlin, Springer-Verlag, 1992.

[26] A.-R. K. RAMAZANOv, On approximation by polynomials and rational functions in Orlicz spaces, Anal. Math. 10 (1984), 117-132.

[27] M. M. RaO AND Z. D. Ren, Theory of Orlicz Spaces, Marcel Dekker, New York, 1991

[28] P. K. Suetin, Series of Faber Polynomials, Gordob and Breack, 1. Reading, 1998.

[29] S. E. WARSCHAWSKI, Über das Ranverhalten der Ableitung der Abbildunggsfunktion bei konformer Abbildung, Math. Z. 35 (1932), 321-456. 